\title{
Data-driven approach to detect common copy-number variations and frequency profiles in a population-based Korean cohort
}

\author{
Sanghoon Moon ${ }^{1,2}$, Young Jin Kim ${ }^{1,2}$, Chang Bum Hong ${ }^{1}$, Dong-Joon Kim ${ }^{1}$, Jong-Young Lee ${ }^{1}$ \\ and Bong-Jo Kim ${ }^{\star, 1}$
}

To date, hundreds of thousands of copy-number variation (CNV) data have been reported using various platforms. The proportion of Asians in these data is, however, relatively small as compared with that of other ethnic groups, such as Caucasians and Yorubas. Because of limitations in platform resolution and the high noise level in signal intensity, in most CNV studies (particularly those using single nucleotide polymorphism arrays), the average number of CNVs in an individual is less than the number of known CNVs. In this study, we ascertained reliable, common CNV regions (CNVRs) and identified actual frequency rates in the Korean population to provide more CNV information. We performed two-stage analyses for detecting structural variations with two platforms. We discovered 576 common CNVRs ( 88 CNV segments on average in an individual), and $87 \%$ (501 of 576) of these CNVRs overlapped by $\geq 1$ bp with previously validated CNV events. Interestingly, from the frequency analysis of CNV profiles, 52 of 576 CNVRs had a frequency rate of $<1 \%$ in the 8842 individuals. Compared with other common CNV studies, this study found six common CNVRs that were not reported in previous CNV studies. In conclusion, we propose the data-driven detection approach to discover common CNVRs including those of unreported in the previous Korean CNV study while minimizing false positives. Through our approach, we successfully discovered more common CNVRs than previous Korean CNV study and conducted frequency analysis. These results will be a valuable resource for the effective level of CNVs in the Korean population.

European Journal of Human Genetics (2011) 19, 1167-1172; doi:10.1038/ejhg.2011.103; published online 6 July 2011

Keywords: common copy-number variation; CNV profile; Asian CNV; structural variation

\section{INTRODUCTION}

A copy-number variation $(\mathrm{CNV})$ is a duplication or deletion of a DNA segment ranging from a kilobase to several megabases and is relatively common and widespread in the human genome. CNVs are also major sources of genomic variation along with single nucleotide polymorphisms (SNPs). ${ }^{1,2}$ In contrast to SNPs, certain CNVs encompass a single gene or a set of genes, and thus duplication or deletion of these CNVs is associated with disease susceptibility and gene dosage. ${ }^{1,2}$ In addition, some CNV studies have reported different profiles of population-specific $\mathrm{CNVs},{ }^{3-14}$ and these stratified $\mathrm{CNV}$ s have been used for further studies of natural selection and human adaptation to environmental pressures. ${ }^{15}$ To perform a study of genomic structure with CNVs, it is essential to accurately ascertain CNVs that consistently occur in the population. ${ }^{15}$

To date, hundreds of thousands of CNVs from large-scale analyses have been reported using various high-resolution genotyping platforms. $^{3-14}$ These data have been deposited in several public databases such as the Database of Genomic Variants (DGVs; http://projects. tcag.ca/variation/) for normal individuals and the Database of Chromosomal Imbalance and Phenotype in Humans using Ensembl Resources (https://decipher.sanger.ac.uk/) for affected individuals.
Although there are ethnic differences among certain CNVs of different populations, in practice the proportion of Asians in these data has been relatively small as compared with that of other ethnic groups, such as Caucasian and Yoruba. Hence, it is necessary to conduct more Asian $\mathrm{CNV}$ studies to understand the genomic loci that account for phenotypic variation and genetic etiology in populations with Asian ancestry.

Recent reports have suggested that common CNVs that can be typed on existing platforms are unlikely to associate with common human diseases. ${ }^{6}$ Despite several successes, ${ }^{16-18}$ common CNVs seem to be nominal genomic markers for studying complex phenotypes. The number of CNVs used for association analyses has been limited by low resolution, platform specificity and the type of CNV class. Most previous studies extracted CNV information from SNP genotyping chips, which are not optimally designed for CNV discovery. Even with CGH chips, which are designed for detecting structural variation, Park et $a l^{7}$ reported that in comparison with CNVs identified in AK1 $\sim 40 \%$ of CNV calls from one specific platform (Agilent 24M aCGH, Santa Clara, CA, USA) were not detectable on the other (NimbleGen $42 \mathrm{M}$ aCGH, Indianapolis, IN, USA) because of platform specificity. Therefore, the discovery of common CNVs is still regarded as a challenging issue for research concerning complex phenotypes.

\footnotetext{
${ }^{1}$ Division of Structural and Functional Genomics, Center for Genome Science, Korea National Institute of Health, Osong, Korea

${ }^{*}$ Correspondence: Dr B-J Kim, Division of Structural and Functional Genomics, Center for Genome Science, Korea National Institute of Health, Osong 363-951, Korea. Tel: +82 43719 8881; Fax: +82 43719 8908; E-mail: kbj6181@cdc.go.kr

${ }^{2}$ These authors contributed equally to this work.

Received 12 August 2010; revised 27 April 2011; accepted 29 April 2011; published online 6 July 2011
} 
The purpose of our study was to discover reliable, common CNV regions (CNVRs) and to examine the frequency of CNVs in the Korean population. To achieve our aim, we performed two-stage analyses for detecting structural variations. Because of the high signal-to-noise ratio of SNP genotyping chips, the considerable number of falsely detected CNVs has made it challenging to construct CNVRs. In the first stage, we thus identified highly reliable CNVRs from 100 individuals with both a NimbleGen HD2 $3 \times 720 \mathrm{~K}$ aCGH assay and the Affymetrix Genome-Wide Human SNP Array 5.0 (Santa Clara, CA, USA). In the second stage, we carried out frequency analyses of the identified CNVRs in 8842 Korean individuals genotyped with the Affymetrix Genome-Wide Human SNP Array 5.0. Based on our results, this is a reliable approach for detecting common CNVRs and surveying ethnically specific CNV patterns.

\section{SUBJECTS AND METHODS}

\section{Study subjects}

To undertake a large-scale genome-wide analysis study, 10038 healthy individuals (aged 40-69 years) who were enrolled in the population-based cohort were genotyped with the Affymetrix Human Genome-Wide Human SNP Array 5.0 as part of the Korean Genome Epidemiology Study (KoGES). Finally, based on genotyping data, 1196 (11.9\%) out of 10038 individuals were excluded in each step of the SNP quality control procedures (sample call rate $\geq 96 \%$, heterozygosity $\geq 30 \%$, gender inconsistency check, exclusion of patients with tumors and population stratification check). This data set was composed of $52.7 \%$ females. ${ }^{19}$ The resulting raw intensity data files (CEL files) from the 8842 samples were applied to create normalized $\log _{2}$ intensity ratios.

\section{Characteristics of CNV genotyping platforms}

Two different types of genotyping platforms were used to detect CNVs: the NimbleGen HD2 3×720K aCGH (dual-channel array platform) and the Affymetrix Genome-Wide Human SNP Array 5.0. (single-channel array platform). The NimbleGen HD2 $3 \times 720 \mathrm{~K}$ aCGH provides more than 720000 probes for the detection of CNVs. Median inter-probe spacing of the backbone is $<5 \mathrm{~kb}$. As additional probes, about 38000 DGV CNVRs and 8599 CNV events (CNVEs) validated by Wellcome Trust Case Control Consortium ${ }^{6}$ were included. The Affymetrix Genome-Wide Human SNP Array 5.0 contains 500568 SNP probes and an additional $420000 \mathrm{CNV}$ probes. Among the CNV probes, $100000 \mathrm{CNV}$ probes were chosen to cover $2000 \mathrm{CNVs}$ identified in the University of California Santa Cruz. Genome Browser database, and the other 320000 probes were evenly distributed across the genome.

\section{Discovery of reliable and common CNVs}

One reason for false calls of SNP chip is spurious CNVs generated from the noise. Most detection tools raise the threshold for detecting true positives and then detect a small number of CNV. To reduce false positive, however, we used the opposite method. We lowered threshold and detected many putative CNVs. Then, we filtered the false calls using genuine CNVR. To build a reliable CNVR map and carry out a frequency analysis, we performed two-stage analyses for detecting structural variations. In the first stage, we adopted a data-driven CNV detection approach using highly reliable CNVRs discovered in 100 individuals with both the NimbleGen HD2 $3 \times 720 \mathrm{~K}$ aCGH assay and the Affymetrix Genome-Wide Human SNP Array 5.0. The 100 individuals were randomly selected from among the 8842 individuals and then were genotyped on the two different platforms using the same DNA isolated from peripheral blood. The Figure 1 and Supplementary Figure S1 show the overall scheme of our CNV analysis. CNV segments detected in each autosomal region from the Affymetrix platform were compared with those from the NimbleGen platform in the same individual. From the NimbleScan v2.5 result of 100 samples, we compiled CNVRs and used them for the genuine CNVR map for further analysis (Supplementary Figure S1). This step was independently conducted for all 100 individuals. Next, we defined both the overlapping region, which occurred in more than two samples among all 100 samples, and a region that was commonly detected in both platforms as a reliable, common

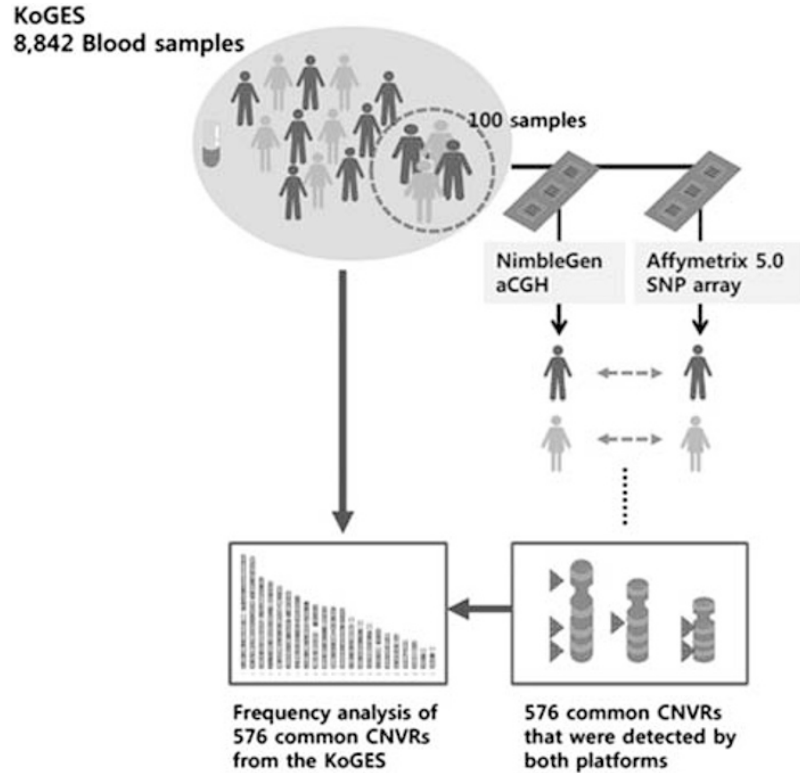

Figure 1 Overall scheme of our CNV analysis. We performed a two-stage analysis to detect structural variations. In the first stage, we discovered highly reliable CNVRs from 100 individuals using two different platforms. The 100 individuals were randomly selected from 8842 individuals from the KoGES and then genotyped on the NimbleGen HD2 $3 \times 720 \mathrm{~K}$ aCGH platform and the Affymetrix Genome-Wide Human SNP Array 5.0 using the same DNA isolated from peripheral blood. In the following stage, we carried out frequency analysis on the discovered 576 CNVRs in 8842 Korean individuals.

CNVR. In the following stage, we carried out a frequency analysis on the discovered CNVRs in the 8842 Korean individuals genotyped with the Affymetrix Genome-Wide Human SNP Array 5.0. (Figure 1 and Supplementary Figure S1). Supplementary Table S1 shows the actual frequency of each of the $576 \mathrm{CNV}$ loci.

Signal intensities were extracted from each platform. For the Affymetrix Genome-Wide Human SNP Array 5.0, pre-processing procedures, such as background subtraction, normalization and summarizing probe set were adopted with the apt-probeset-summarize application (Affymetrix Power Tools; http://www.affymetrix.com/partners_programs/programs/developer/tools/ powertools.affx). After pre-processing procedures, the signal intensity ratio between the test and reference samples (two replicates of NA10851 (Coriell), which was from the HapMap cell line DNA) of each probe was transformed to $\log _{2}$ scale with the chromosomal coordinates of the probes (University of California Santa Cruz version hg18/NCBI Build 36). CNV segments were called using the Genome Alteration Detection Analysis (GADA) segmentation algorithm. ${ }^{20}$ To define the threshold, different threshold Ts were tested from 3 to 8 . Finally, we ran the GADA R-package on the 8842 individuals with $T=3.5$, $\alpha=0.2$ and MinSegLen $=6$, which is a relaxed threshold to maximize the number of multiple matches with CNV segments from the NimbleGen platform. For the CNV detection results from two reference replicates (both generated from NA10851 cells), we selected the one with more matches. For the NimbleGen HD2 $3 \times 720 \mathrm{~K}$, all 100 samples passed experimental control metrics, such as chromosome X shift and mad.1dr with NimbleScan v2.5. After quality control procedures, signal intensities for each probe were also extracted from the test and NA10851 cell line DNA. The threshold for defining CNV segments was set to an average $\log _{2}$ ratio of \pm 0.3 .

\section{Validation of CNVRs by TaqMan copy-number assays}

To assess the CNV calling of false-positive CNVRs, we randomly selected $20 \mathrm{CNV}$ loci from the 576 CNVRs (13 gains and 7 loss loci) for validation. Table 1 shows characteristics of the 20 validated CNVRs. For each CNVR, we carried out TaqMan Copy Number Assays (Applied Biosystems, Foster City, CA, USA) with 20 predesigned primers. All experiments were replicated three times to enhance the validation accuracy. 
Table 1 Validated CNVRs from TaqMan copy number assays

\begin{tabular}{lrrclrrr}
\hline Chromosome & Start & Stop & Length & State & $T P^{a}$ & $F P^{b}$ & PPV \\
\hline Chr1 & 88868282 & 89251066 & $383 \mathrm{~kb}$ & Gain & 100 & 50 & 0.667 \\
Chr2 & 159668015 & 159669082 & $1 \mathrm{~kb}$ & Gain & 25 & 5 & 0.833 \\
Chr3 & 163996134 & 164103516 & $107 \mathrm{~kb}$ & Gain & 29 & 1 & 0.967 \\
Chr4 & 61621813 & 61624741 & $2.9 \mathrm{~kb}$ & Gain & 30 & 0 & 1.000 \\
Chr4 & 186678748 & 186681053 & $2.3 \mathrm{~kb}$ & Gain & 28 & 2 & 0.933 \\
Chr6 & 54037057 & 54041872 & $4.8 \mathrm{~kb}$ & Loss & 30 & 0 & 1.000 \\
Chr6 & 79577799 & 79581568 & $3.8 \mathrm{~kb}$ & Loss & 30 & 0 & 1.000 \\
Chr6 & 74647117 & 74656877 & $9.8 \mathrm{~kb}$ & Loss & 124 & 26 & 0.827 \\
Chr7 & 22401397 & 22403203 & $1.8 \mathrm{~kb}$ & Gain & 22 & 8 & 0.733 \\
Chr7 & 70058931 & 70063735 & $4.8 \mathrm{~kb}$ & Gain & 27 & 3 & 0.900 \\
Chr8 & 112363280 & 112365400 & $2.1 \mathrm{~kb}$ & Loss & 25 & 5 & 0.833 \\
Chr9 & 70927986 & 70933108 & $5.1 \mathrm{~kb}$ & Gain & 28 & 2 & 0.933 \\
Chr9 & 23348479 & 23367652 & $19.2 \mathrm{~kb}$ & Loss & 117 & 33 & 0.780 \\
Chr10 & 31483686 & 31484714 & $1 \mathrm{~kb}$ & Gain & 21 & 9 & 0.700 \\
Chr13 & 37955433 & 37958105 & $2.7 \mathrm{~kb}$ & Gain & 21 & 9 & 0.700 \\
Chr13 & 49967437 & 49970106 & $2.7 \mathrm{~kb}$ & Loss & 29 & 1 & 0.967 \\
Chr15 & 60493430 & 60494943 & $1.5 \mathrm{~kb}$ & Gain & 28 & 2 & 0.933 \\
Chr18 & 61917841 & 61920121 & $2.3 \mathrm{~kb}$ & Loss & 26 & 4 & 0.867 \\
Chr20 & 1337680 & 1338783 & $1.1 \mathrm{~kb}$ & Gain & 26 & 4 & 0.867 \\
Chr21 & 43794757 & 43797350 & $2.6 \mathrm{~kb}$ & Gain & 27 & 3 & 0.900 \\
\hline
\end{tabular}

Abbreviations: ${ }^{\mathrm{a}} \mathrm{TP}$ : true positive; ${ }^{\mathrm{b}} \mathrm{FP}$, false positive; ${ }^{\mathrm{C} P P V}$, positive predictive value. PPV: no. of TP/(no. of TP+no. of FP) 100 .
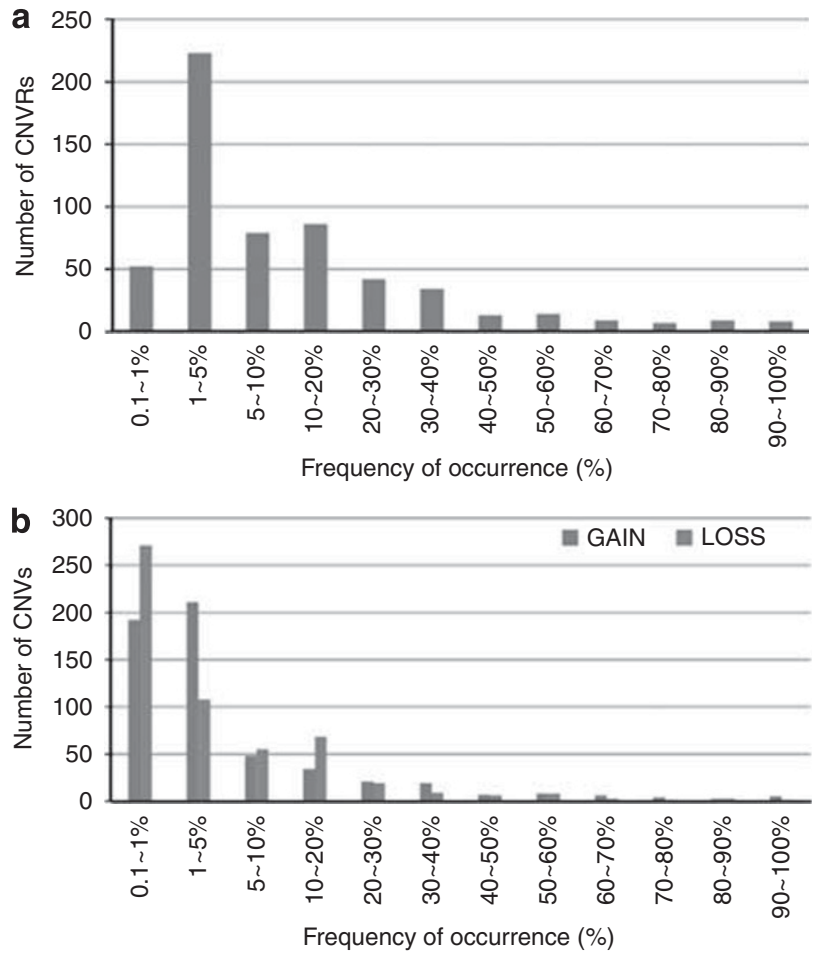

Figure 2 CNV frequency rates for the 576 detected CNVRs in 8842 Korean individuals. (a) We surveyed the frequency rate of each region. Of the 576 CNVRs, 223 had frequency rates of $1-5 \%$, and 52 CNVRs had frequency rates of $<1 \%$ (rare variant). (b) Frequency rates of the detected CNVRs were divided into gains and losses.

\section{RESULTS}

Discovery of common CNVRs

Comparing autosomal CNV segments detected in the Affymetrix and NimbleGen platforms for 100 randomly selected samples, we found an

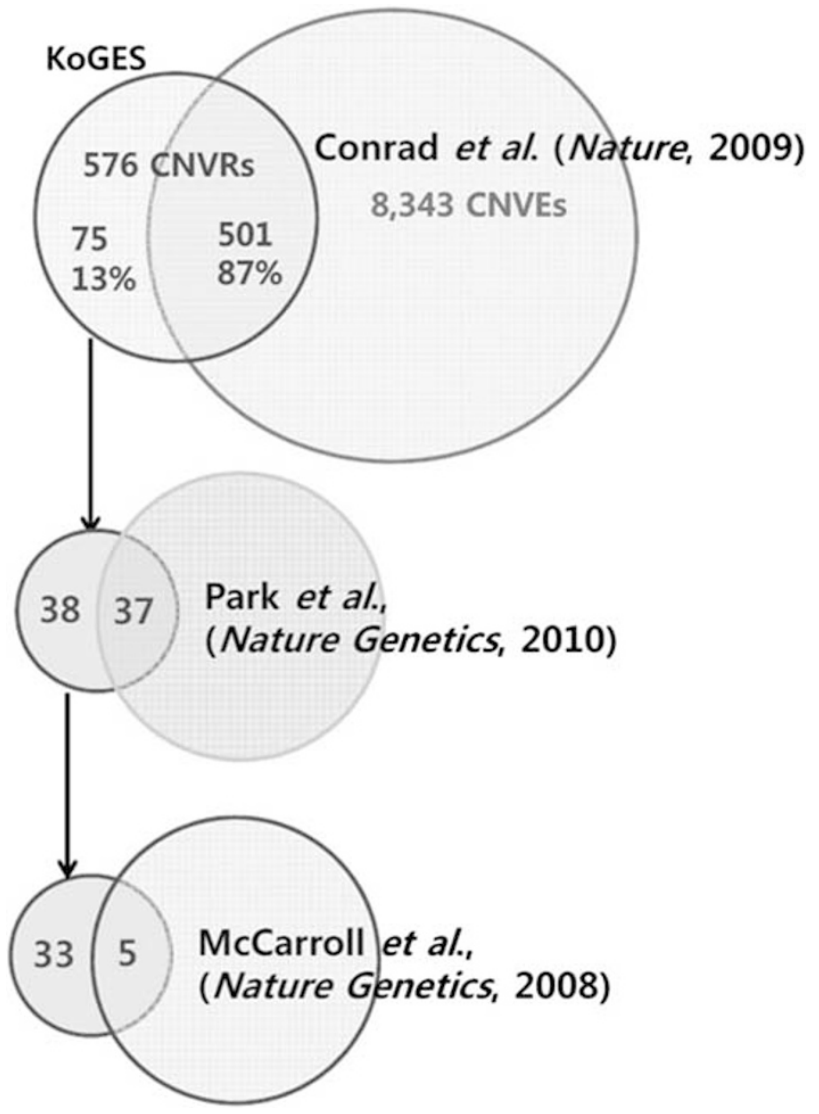

Figure 3 Comparison of results with previously reported common CNVRs. Detected CNVRs (576) were compared with three well-defined CNVR studies using three kinds of platforms. Most $(87 \% ; 501$ of 576$)$ of the detected CNVs overlapped with 8343 autosomal CNVEs with the NimbleGen platform. ${ }^{6}$ Regarding the remainder (75 of 576), 37 CNVRs overlapped with the results of a study examining Asian common CNVRs with the Agilent platform. ${ }^{7}$ For the 38 non-overlapping CNVRs, we examined their concurrence with the common CNVRs from 270 HapMap samples with the Affymetrix platform. ${ }^{3}$ Most (33 of 38 ) did not concur with the results of McCarroll et al. ${ }^{3}$

average of $88 \mathrm{CNV}$ segments in each individual. In total, 8779 segments detected in the 100 individuals were also assigned to CNVRs. Among these segments, the data-driven approach identified 807 common CNVRs that recurred in more than two samples of all 100 samples. Finally, 576 autosomal CNVRs ranging in length from $1 \mathrm{~kb}$ to $4.56 \mathrm{Mb}$ were selected (Supplementary Table S1 and Supplementary Figure S3). The mean and median length of these CNVRs was 12.6 and $113 \mathrm{~kb}$, respectively (Supplementary Table S4). Supplementary Table S3 and supplementary Figure S4 show the number of CNVs that affected genes. The median and the mean number of probe included in break points was 33.5 and 69.5, respectively (Supplementary Figure S2). Furthermore, we examined the CNV state of each of the 576 regions to determine whether it was a copy-number gain, loss or complex (gain and loss). Moreover, the CNV type of the 576 CNVRs was also determined to be either common or rare by the $1 \%$ frequency rate.

Frequency analysis of CNVRs

To establish CNV profiles, we examined the frequency of occurrence in our detected CNVRs by studying their occurrence in the total cohort 
of 8842 individuals screened onto Affymetrix 5.0. arrays. Figure 2 shows the distribution of the 576 CNVRs by frequency rate. Most (91\%; 524 of 576 CNVRs) of the detected CNVRs had a $>1 \%$ frequency rate in the 8842 individuals. We suggest that these results support our detection strategy for common CNVs and that there may be additional common CNVRs in Koreans. Interestingly, the majority of the 576 CNVRs (223 regions) had frequency rates of $1-5 \%$. Interestingly, 47 regions ( $8 \%$ of all CNVRs detected) varied in more than $50 \%$ of all 100 cases.

\section{Comparison with previously reported common CNVRs}

Figure 3 shows results from the cascade approach comparing our CNVRs with previously reported common CNVRs. Most (87\%; 501 of 576) of the total detected CNVRs overlapped by $\geq 1$ bp with 8343 autosomal CNVEs. ${ }^{6}$ Comparing the 501 overlapping CNVRs with the results of Conrad et al, ${ }^{6} 51$ of these CNVRs had a frequency rate of $<1 \%$. Of the remainder ( 75 of 576 ), only 37 CNVRs overlapped with the results of common Asian CNVRs including 10 Korean samples genotyped on the Agilent $24 \mathrm{M}$ platform. ${ }^{7}$ Concerning the 38 nonoverlapping CNVRs, we examined their concurrence with common
CNVRs from 270 HapMap samples genotyped on the Affymetrix Human Genome-wide Human SNP array 6.0. ${ }^{3}$ Most (33 of 38) did not concur with the results of McCarroll et al. ${ }^{3}$

We compared these 33 regions with all CNVRs listed in the DGV in more detail (Table 2). Most CNVRs (26 of 33) overlapped with either CNVs or CNVRs from 23 different CNV studies. ${ }^{4-6,9-14,21-35}$ In particular, 12 of 33 CNVRs matched CNVEs identified by Conrad et $a l,{ }^{6}$ and these were not listed as validated CNVEs. Consequently, we found seven unlisted CNVRs in the DGV. However, when the 33 CNVRs were compared with the CNVRs described by Yim et al, ${ }^{8}$ eight regions overlapped. Interestingly, one region among the eight CNVRs overlapped with the seven unlisted CNVRs. In total, we found six common, previously unreported CNVRs. When we compared our CNVRs with union set of CNVRs from four different studies, we also observed the same results as those of cascade approach.

\section{Gene ontology analysis of CNVRs}

We surveyed RefSeq genes partially or entirely encompassing 576 CNVRs and found 629 RefSeq genes. To assess the functional

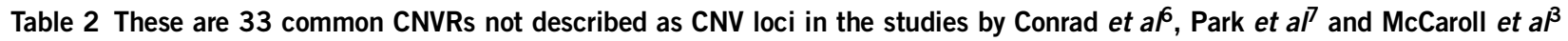

\begin{tabular}{|c|c|c|c|c|c|}
\hline \multirow[t]{2}{*}{ Chr } & \multirow[t]{2}{*}{ Start } & \multirow[t]{2}{*}{ End } & \multirow[t]{2}{*}{ References for overlapping CNVs in DGV } & \multicolumn{2}{|c|}{ Frequency (\%) } \\
\hline & & & & Gain & Loss \\
\hline Chr2a & 3803336 & 3952526 & McKernan et $a^{R 1}$ & 79.9 & 0.1 \\
\hline Chr2 & 223468381 & 223471352 & Bentley et $a l^{22}$ Korbel et al, ${ }^{23}$ Levy et al, ${ }^{24}$ McKernan et al, ${ }^{21}$ Wang et al, ${ }^{11}$ Wheeler et $a{ }^{25}$ & 0.0 & 1.3 \\
\hline $\mathrm{Chr}^{\mathrm{a}}$ & 4234309 & 4252262 & Itsara et al, ${ }^{10}$ Redon et al, ${ }^{4}$ Shaikh et al, ${ }^{9}$ Zogopoulos et al26 & 0.5 & 18.1 \\
\hline Chr4 & 190062985 & 190128452 & Conrad et al, ${ }^{6}$ Zogopoulos et aR ${ }^{26}$ & 1.0 & 0.7 \\
\hline Chr5 & 1785959 & 1822301 & No overlapping CNVs described, indel, inversion & 18.0 & 0.0 \\
\hline Chr7a & 11278981 & 11314260 & Conrad et al, ${ }^{27}$ Matsuzaki et al, ${ }^{28}$ Redon et al & 0.1 & 13.6 \\
\hline Chr8a & 92185630 & 92249955 & Mckernan et al, ${ }^{21}$ Pinto et al, ${ }^{29}$ Redon et al & 0.4 & 21.4 \\
\hline Chr8 & 145820725 & 145821995 & Wong et al ${ }^{14}$ & 3.1 & 0.0 \\
\hline Chr9 & 38189356 & 38215032 & Redon et $a l_{,}^{4}$ Wong et al ${ }^{14}$ & 26.5 & 0.0 \\
\hline Chr9 & 136496422 & 136511685 & Conrad et al, ${ }^{6}$ Levy et $a,^{24}$ Wang et $a l,{ }^{30}$ Wong et all & 20.5 & 5.7 \\
\hline Chr9 & 136881046 & 136882910 & Conrad et a/6 & 3.6 & 0.0 \\
\hline Chr10 & 134015916 & 134026670 & Conrad et $a l^{6}{ }^{6}$ Itsara et al ${ }^{10}$ & 4.1 & 0.1 \\
\hline Chr11 & 45080630 & 45089496 & No overlapping CNVs described, indel & 22.2 & 0.0 \\
\hline Chr12 & 131955341 & 131960337 & McKernan et al, 21 Matsuzaki et aR8 & 1.8 & 0.1 \\
\hline Chr13a & 54839168 & 54850687 & No overlapping CNVs described, indel & 0.1 & 11.5 \\
\hline Chr15 & 83262590 & 83272701 & Itsara et al, ${ }^{10}$ Jakobsson et $a^{\beta 1}$ & 1.6 & 0.1 \\
\hline Chr17a & 113372 & 119301 & Kidd et $a l^{5}$ & 7.1 & 0.0 \\
\hline Chr17 & 2054397 & 2149701 & No overlapping CNVs described, indel & 0.4 & 16.0 \\
\hline Chr17a & 78371877 & 78373883 & Conrad et $a^{6}$ & 2.8 & 0.1 \\
\hline Chr18 & 75410941 & 75413108 & Ahn et $a l^{32}$ Conrad et al, ${ }^{6}$ Levy et al, ${ }^{24}$ Wang et al, ${ }^{11}$ Wong et al ${ }^{14}$ & 2.5 & 0.7 \\
\hline Chr18 & 75667308 & 75669900 & Conrad et $a^{\beta}$ & 2.6 & 0.3 \\
\hline Chr19 & 311044 & 312669 & Conrad et al, 6 Mills et al, ${ }^{33}$ Perry et al, ${ }^{12}$ Redon et a/ & 1.8 & 0.2 \\
\hline Chr19 & 355959 & 374597 & Conrad et $a l,{ }^{6}$ Jakobsson et $a l,{ }^{31}$ Wang et $a{ }^{\beta 0}$ & 1.8 & 0.2 \\
\hline Chr19 & 985108 & 996171 & Conrad et al, ${ }^{6}$ Locke et al, ${ }^{34}$ McKernan et al, ${ }^{21}$ Perry et al, ${ }^{12}$ Wong et al ${ }^{14}$ & 1.6 & 0.2 \\
\hline Chr19 & 1578740 & 1580117 & Conrad et $a l^{6}{ }^{6}$ de Smith et $a l^{13}$ & 1.8 & 0.2 \\
\hline Chr19 & 5201759 & 5219582 & No overlapping CNVs described, indel & 1.6 & 0.0 \\
\hline Chr19 & 44503723 & 44527789 & Conrad et a 6 & 1.2 & 0.0 \\
\hline Chr19 & 48834740 & 48836125 & Conrad et a/6 & 0.9 & 0.0 \\
\hline Chr19a & 58605551 & 58633953 & $\begin{array}{l}\text { Ahn et al, }{ }^{32} \text { Itsara et al, }{ }^{10} \text { Jakobsson et al, }{ }^{31} \text { Matsuzaki et } a l,{ }^{28} \text { McKernan et al, }{ }^{21} \text { Redon et al, } \\
\text { Wang et } a{ }^{30}\end{array}$ & 1.0 & 2.8 \\
\hline Chr20 & 6917697 & 6923002 & No overlapping CNVs described, indel & 0.0 & 2.8 \\
\hline Chr20 & 61310499 & 61325605 & Itsara et al, ${ }^{10}$ Jakobsson et al, ${ }^{31} \mathrm{Kim}$ et al, ${ }^{35}$ Perry et al, ${ }^{12}$ Shaikh et al, ${ }^{9}$ Wang et al, ${ }^{30}$ Wong et al14 & 2.7 & 0.4 \\
\hline Chr21 & 42147413 & 42153194 & No overlapping CNVs described & 1.0 & 0.2 \\
\hline Chr21 & 45224142 & 45226832 & de Smith et $a{ }^{13}{ }^{13}$ Redon et aA & 4.4 & 0.0 \\
\hline
\end{tabular}

${ }^{a}$ CNVRs overlapping with those of Yim et $a l^{8}$. 
Table 3 Gene ontology analysis results of genes overlapping with 576 CNVRs using the DAVID functional annotation tool

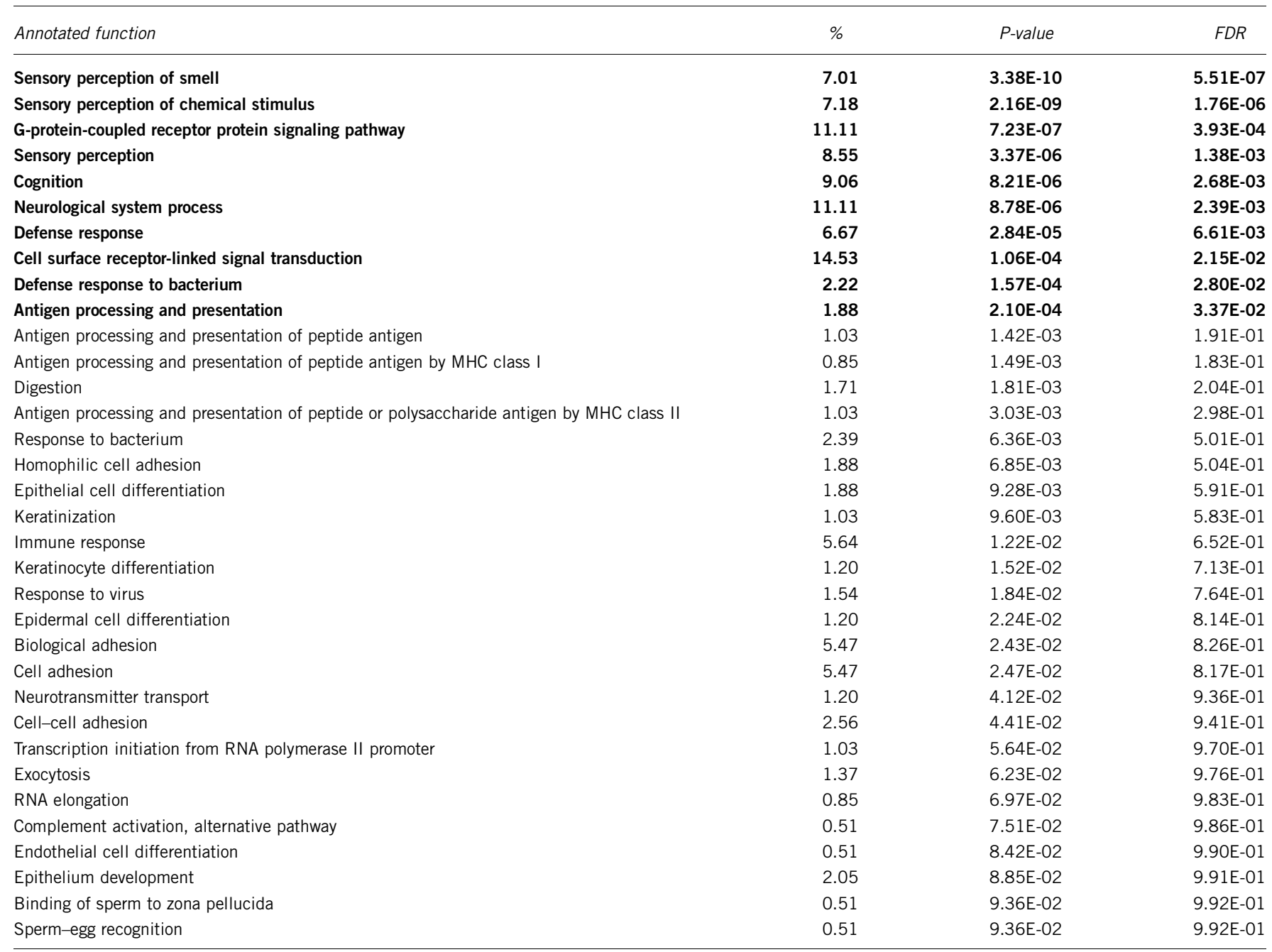

Abbreviation: FDR, false discovery rate (Benjamini and Hochberg method).

FDRs that are $<0.05$ are shown in boldface.

implications of these CNVRs, we conducted gene ontology analysis using the Database for Annotation, Visualization and Integrated Discovery (DAVID) functional annotation tool ${ }^{36}$ and the PANTHER classification system. ${ }^{37}$ Table 3 and Supplementary Table S2 show gene ontology results from these two analysis tools. Genes involved in sensory perception, cognition, neurological system processes, defense responses and immune responses were mainly included in the DAVID results. Similarly, PANTHER results showed genes involved in metabolic processes, cellular communication, immune system processes and responses to stimuli.

\section{Assessment of accuracy of detected CNVRs by experimental validation}

To assess the accuracy for our CNV calling strategy, we carried out TaqMan Copy Number Assays (Applied Biosystems) on 20 randomly selected CNV loci among the 576 CNVRs. Table 1 shows characteristics of the 20 validated CNVRs. As a result of our experiment, we defined the positive predictive value (PPV) as the proportion of CNVs with positive test results, which were correctly predicted. And we applied this statistic as the measurement standard of accuracy. The average PPV of our validation test was 0.886 (Table 1).

\section{DISCUSSION}

The high noise level of the signal intensity is one of the drawbacks of CNV studies, especially those that use SNP arrays. It is not easy to filter out noise during the detection process. To dissect noise from signals, a parameter or a cutoff on the CNV calling algorithm is changed repeatedly to select an optimal value. During this process, many legitimate signals are poorly considered, and thus a significant number of legitimate samples are pruned. For example, although 670 and $1098 \mathrm{CNVs}$ on average in an individual were reported from two previous studies using an ultra high-resolution array, in most CNV studies using SNP arrays, the number of average CNVs in each individual has not exceeded $50 .^{8-10,30,38}$

Recently, Yim et $a l^{8}$ discovered a set of CNVRs from 3578 of the 8842 under the KoGES samples. However, they demonstrated 40.3 CNVs per genome after analyzing 3578 individuals. Despite the fact that a subset of identical samples was used for the analysis, only a few common CNVs were detected because of the limited capability of SNP genotyping chips and the stringent threshold criteria for $\mathrm{CNV}$ calling. In practice, Yim et al ${ }^{8}$ reported 656 common CNVRs among 4003 CNVRs in their study. 
In our current study, we performed the data-driven detection approach to discover common CNVRs including those of previously unreported in Yim et $a l^{8}$ and then confirmed the frequency rates in these regions using more expanded samples than Yim et al. ${ }^{8}$

We also compared our results with previously reported common CNVRs. In all, $87 \%$ of detected CNVs (501 of 576) overlapped with previously validated CNVEs by Conrad et al. ${ }^{6}$ However, in comparison with previously reported common Asian $\mathrm{CNVs}^{7}$ to determine whether the discrepancies originated from Korean-specific CNVs, $64 \%$ of these CNVs (371 of 576) were shared in each study. We believe that platform-dependent CNVs may result in a lower match rate than in Conrad et $a^{6}$ because the NimbleGen HD2 $3 \times 720 \mathrm{~K}$ aCGH assay contains targeted $\mathrm{CNV}$ probes for the 8599 CNVEs described by Conrad et al (see characteristics of CNV genotyping platforms in the Subjects and methods). ${ }^{6}$ Consequently, we found six previously unreported CNVRs. However, we cannot conclude that these CNVRs are Korean specific because of the highly variable discrepancy among genotyping platforms.

We then surveyed the frequency rate of each region and determined whether the region was actually common in the population. As a result, 223 of 576 CNVRs had frequency rates of $1-5 \%$, and 47 CNVRs occurred in $>50 \%$ of individuals. Interestingly, 52 of 576 CNVRs had a frequency rate of $<1 \%$ in 8842 individuals. Moreover, 51 of 52 regions overlapped with those reported by Conrad et $a l,{ }^{6}$ as described above.

There are some limitations associated with our approach. First, as we mainly focused on common CNVRs, we could not address the presence of rare CNVs. Second, despite our efforts to solve the noise problem, we still ascertained only a part of the CNVs. Thus, further studies are required to complete the Korean CNV profile.

Nevertheless, we propose a reliable approach for detecting common CNVRs. As only 44\% (251 of 576) of CNVRs matched those of Yim et $a l,{ }^{8}$ the CNVRs from the two studies are complementary to each other. Therefore, further analysis combining the data from Yim et al ${ }^{8}$ and the data in our study will be a substantial resource for mapping structural variants in the Korean population. Moreover, such a study will give an extended map of $\mathrm{CNV}$ markers and contribute to create an association map of genomic loci that accounts for the variation of thousands of phenotypes available in the KoGES.

\section{CONFLICT OF INTEREST}

The authors declare no conflict of interest.

\section{ACKNOWLEDGEMENTS}

This work was supported by an intramural grant from the Korea National Institute of Health (2009-N00469-00, 2010-N73001-00) and grants from Korea Centers for Disease Control and Prevention (4845-301, 4851-302, 4851-307). We are grateful to Professor Yeun-Jun Chung of the Integrated Research Center for Genome Polymorphism, Catholic University of Korea, for making two replicates of reference genotype data (NA10851) available.

1 Freeman JL, Perry GH, Feuk L et al: Copy number variation: new insights in genome diversity. Genome Res 2006; 16: 949-961.

2 Choy KW, Setlur SR, Lee $C$ et al: The impact of human copy number variation on a new era of genetic testing. BJOG 2010; 117: 391-398.

3 McCarroll SA, Kuruvilla FG, Korn JM et al: Integrated detection and population-genetic analysis of SNPs and copy number variation. Nat Genet 2008; 40: 1166-1174.

4 Redon R, Ishikawa S, Fitch KR et al: Global variation in copy number in the human genome. Nature 2006; 444: 444-454.
5 Kidd JM, Cooper GM, Donahue WF et al: Mapping and sequencing of structural variation from eight human genomes. Nature 2008; 453: 56-64.

6 Conrad DF, Pinto D, Redon R et al: Origins and functional impact of copy number variation in the human genome. Nature 2009; 464: 704-712.

7 Park H, Kim J, Ju YS et al: Discovery of common Asian copy number variants using integrated high-resolution array CGH and massively parallel DNA sequencing. Nat Genet 2010; 42: 400-405.

8 Yim SH, Kim TM, Hu HJ et al: Copy number variations in East-Asian population and their evolutionary and functional implications. Hum Mol Genet 2010; 19: 1001-1008.

9 Shaikh TH, Gai X, Perin JC et al: High-resolution mapping and analysis of copy number variations in the human genome: a data resource for clinical and research applications. Genome Res 2009; 19: 1682-1690.

10 Itsara A, Cooper GM, Baker C et al: Population analysis of large copy number variants and hotspots of human genetic disease. Am J Hum Genet 2009; 84: 148-161.

11 Wang J, Wang W, Li R et al: The diploid genome sequence of an Asian individual. Nature 2008; 456: 60-65.

12 Perry GH, Ben-Dor A, Tsalenko A et al: The fine-scale and complex architecture of human copy-number variation. Am J Hum Genet 2008; 82: 685-695.

13 de Smith AJ, Tsalenko A, Sampras N et al: Array CGH analysis of copy number variation identifies 1284 new genes variant in healthy white males: implications for association studies of complex disease. Hum Mol Genet 2007; 16: 2783-2794.

14 Wong KK, deLeeuw RJ, Dosanjh NS et al: A comprehensive analysis of common copynumber variations in the human genome. Am J Hum Genet 2007; 80: 91-104.

15 Wu LY, Chipman HA, Bull SB et al: A Bayesian segmentation approach to ascertain copy number variations at the population level. Bioinformatics 2009; 25: 1669-1679.

16 Gonzalez E, Kulkarni $H$, Bolivar $H$ et al: The influence of CCL3L1 genecontaining segmental duplications on HIV-1/AIDS susceptibility. Science 2005; 307: 1434-1440.

17 Fanciulli M, Norsworthy PJ, Petretto E et al: FCGR3B copy number variation is associated with susceptibility to systemic, but not organ-specific autoimmunity. Nat Genet 2007; 39: 721-723.

18 Perry GH, Dominy NJ, Claw KG et al: Diet and the evolution of human amylase gene copy number variation. Nat Genet 2007; 39: 1256-1260.

19 Cho YS, Go MJ, Kim YJ et al: A large-scale genome-wide association study of Asian populations uncovers genetic factors influencing eight quantitative traits. Nat Genet 2009; 41: 527-534.

20 Pique-Regi R, Monso-Varona J, Ortega A, Seeger RC, Triche TJ, Asgharzadeh S: Sparse representation and Bayesian detection of genome copy number alterations from microarray data. Bioinformatics 2008; 24: 309-318.

21 McKernan KJ, Peckham HE, Costa GL et al: Sequence and structural variation in a human genome uncovered by short-read, massively parallel ligation sequencing using two-base encoding. Genome Res 2009; 19: 1527-1541.

22 Bentley DR, Balasubramanian S, Swerdlow HP et al: Accurate whole human genome sequencing using reversible terminator chemistry. Nature 2008; 456: 53-59.

23 Korbel JO, Urban AE, Affourtit JP et al: Paired-end mapping reveals extensive structural variation in the human genome. Science 2007; 318: 420-426.

24 Levy S, Sutton G, Ng PC et al: The diploid genome sequence of an individual human. PLOS Biol 2007; 5: 3254

25 Wheeler DA, Srinivasan M, Egholm M et al: The complete genome of an individual by massively parallel DNA sequencing. Nature 2008; 452: 872-876.

26 Zogopoulos G, Ha KC, Naqib F et al: Germ-line DNA copy number frequencies in a large North American population. Hum Genet 2007; 122: 345-353.

27 Corad DF, Andrews TD, Carter NP, Hurles ME, Pritchard JK: A high-resolution survey of deletion polymorphism in the human genome. Nat Genet 2005; 38: 75-81.

28 Matsuzaki H, Wang PH, Hu J, Rava R, Fu GK: High resolution discovery and confirmation of copy number variants in 90 Yoruba Nigerians. Genome Biol 2009; 10: R125.

29 Pinto D, Marshall C, Feuk L, Scherer SW: Copy-number variation in control population cohorts. Hum Mol Genet 2007; 16: R168-R173.

30 Wang K, Li M, Hadley D et al: PennCNV: an integrated hidden Markov model designed for high-resolution copy number variation detection in whole-genome SNP genotyping data. Genome Res 2007; 17: 1665-1674.

31 Jakobsson M, Scholz SW, Scheet $\mathrm{P}$ et al: Genotype, haplotype and copy-number variation in worldwide human populations. Nature 2008; 451: 998-1003.

32 Ahn SM, Kim TH, Lee S et al: The first Korean genome sequence and analysis: full genome sequencing for a socio-ethnic group. Genome Res 2009; 19: 1622-1629.

33 Mills RE, Luttig CT, Larkins et al: An initial map of insertion and deletion (INDEL) variation in the human genome. Genome Res 2006; 16: 1182-1190.

34 Locke DP, Sharp AJ, McCarroll SA et al: Linkage disequilibrium and heritability of copynumber polymorphisms within duplicated regions of the human genome. Am J Hum Genet 2006; 79: 275-290.

$35 \mathrm{Kim}$ J, Ju YS, Park $\mathrm{H}$ et al: A highly annotated whole-genome sequence of a Korean individual. Nature 2009; 460: 1011-1015.

36 Huang DW, Sherman BT, Lempicki RA: Systematic and integrative analysis of large gene lists using DAVID Bioinformatics Resources. Nat Protoc 2009; 4: 44-57.

$37 \mathrm{Mi} \mathrm{H}$, Dong Q, Muruganujan A, Gaudet P, Lewis S, Thomas PD: PANTHER version 7: improved phylogenetic trees, orthologs and collaboration with the Gene Ontology Consortium. Nucleic Acids Res 2010; 38: D204-D210.

38 Glessner JT, Wang K, Cai G et al: Autism genome-wide copy number variation reveals ubiquitin and neuronal genes. Nature 2009; 459: 569-573.

Supplementary Information accompanies the paper on European Journal of Human Genetics website (http://www.nature.com/ejhg) 